\title{
Enhanced recovery programmes can also reduce postoperative mortality
}

\author{
Karem Slim ${ }^{1,2}$ (D) Jean Joris ${ }^{1,3} \cdot$ Laurent Delaunay ${ }^{1,4}$ \\ Received: 3 February 2021 / Accepted: 8 February 2021 / Published online: 18 February 2021 \\ (C) The Author(s), under exclusive licence to Springer-Verlag GmbH, DE part of Springer Nature 2021
}

Fewer postoperative complications and shorter length of hospital stay are amply demonstrated benefits of enhanced recovery programmes (ERPs) across surgical specialties reported in the literature. In recent years, an increasing number of reports suggest that ERP may have further benefits. We highlighted that improved 5-year survival after colorectal cancer surgery could be an additional benefit of ERPs if more than $70 \%$ of the ERP measures were implemented [1]. The underlying mechanism is probably multifactorial: better nutritional support, better analgesia, less postoperative morbidity, prompter initiation of adjuvant treatment, and reduced perioperative immune dysfunction. But besides improved long-term survival after cancer surgery, can ERPs also reduce short-term postoperative mortality?

Reductions in postoperative 30-day mortality by ERPs have been described in cases of orthopaedic surgery, in cohort studies and meta-analysis [2, 3]. In the study by Khan et al. on the first 6000 hip and knee arthroplasties, reduction in mortality was especially significant when there were more comorbidities (hypertension, diabetes, chronic obstructive pulmonary disease) in the ERP group [2]. The meta-analysis by Deng et al., including nearly 17,000 patients, confirmed these results with an effect size of around $50 \%$ and without heterogeneity $\left(I^{2}=10 \%\right)$. The benefit of ERPs was observed, in this meta-analysis, mainly in the 60-70-year age group [3]. However, most of these studies did not use a contemporary

Karem Slim

kslim@chu-clermontferrand.fr

1 Francophone Group for Enhanced Recovery After Surgery (GRACE), Beaumont, France

2 Department of Digestive Surgery, University Hospital, CHU, Clermont-Ferrand, France

3 Department of Anaesthesia, University Hospital Liege, Liege, Belgium

4 Department of Anaesthesia, General Clinic, Annecy, France control group. An impact on postoperative mortality of the progressive widespread adoption of simple perioperative measures (posterior surgical approach for hip arthroplasty, thromboprophylaxis, and spinal anaesthesia) [4], now parts of ERPs, was indeed observed in the early 2000s, just before the formal ERP era. ERPs are no more no less than the logical outcome of all the advances in care.

Does ERP influence mortality after types of surgery other than orthopaedic surgery? This question was addressed very recently in an American study including nearly 9500 patients who underwent major orthopaedic, but also hepatobiliary, colorectal, gynaecological, bariatric, or urological surgery [5]. This before-after study reported that the 30-day mortality decreased from $0.6 \%$ before ERP to $0.2 \%(p=0.002)$ with ERP, and 1-year mortality decreased from 5.1 to $3.9 \%(p<0.0001)$. These benefits persisted 3 years after surgery. Surprisingly decreased mortality was observed despite reported minimalist implementation of ERP measures (minimally invasive surgery, early refeeding and mobilisation, early removal of urinary drainage, prevention of nausea and vomiting, perioperative multimodal analgesia). Such basic (or light) ERP implementation is questionable. The adherence to more than $70 \%$ of the elements of the protocol is however usually required to experience less postoperative complication rate and shorten the duration of hospitalisation. Other items of ERP such as patient information and active participation, preoperative nutritional support, limited fasting, and no or limited drainage were probably implemented routinely before ERPs were launched in the centres participating in the study. We think that such revolutionary and promising results call for optimal adherence to ERP. The design of the large American study [5] is interesting, since it involved multiple specialties and multiple hospitals (both academic and community ones) and especially a rolling implementation of ERPs, making the respective groups (ERP vs. control groups) quasi-contemporary across the hospitals. This design improves the level of evidence and overcomes the limitation of before-after studies conducted over several years. The only obvious limitation of 
this study is that the results were reported without a subgroup analysis by surgical specialty. The external validity for each specialty thus remains limited.

In conclusion, we are now probably beyond the era of "ERPs are feasible without increasing readmission and postoperative mortality". The initial benefits of ERPs are extended to more and more surgical settings and to new fields. According to these recent reports, ERP favourably affect postoperative mortality. Large nationwide studies should be launched to confirm this additional benefit of ERPs.

\section{References}

1. Slim K, Regimbeau JM, Francophone Group for Enhanced Recovery After Surgery (GRACE) (2018 Jun) Increased survival might be an unexpected additional advantage of enhanced recovery after surgery programs. J Visc Surg. 155(3):169-171. https://doi.org/ 10.1016/j.jviscsurg.2018.02.006

2. Khan SK, Malviya A, Muller SD, Carluke I, Partington PF, Emmerson KP, Reed MR (2014 Feb) Reduced short-term complications and mortality following enhanced recovery primary hip and knee arthroplasty: results from 6,000 consecutive procedures. Acta Orthop. 85(1):26-31. https://doi.org/10.3109/ 17453674.2013.874925

3. Deng QF, Gu HY, Peng WY, Zhang Q, Huang ZD, Zhang C, Yu YX (2018 Dec) Impact of enhanced recovery after surgery on postoperative recovery after joint arthroplasty: results from a systematic review and meta-analysis. Postgrad Med J. 94(1118):678-693. https:// doi.org/10.1136/postgradmedj-2018-136166

4. Hunt LP, Ben-Shlomo Y, Clark EM, Dieppe P, Judge A, MacGregor AJ, Tobias JH, Vernon K (2013) Blom AW; National Joint Registry for England, Wales and Northern Ireland. 90-day mortality after 409, 096 total hip replacements for osteoarthritis, from the National Joint Registry for England and Wales: a retrospective analysis. Lancet. 382(9898):1097-1104. https://doi.org/10.1016/S0140-6736(13) 61749-3

5. Esper SA, Holder-Murray J, Subramaniam K, Boisen M, Kenkre TS, Meister K, Foos S, Wong H, Howard-Quijano K, Mahajan A (2020) Enhanced recovery protocols reduce mortality across eight surgical specialties at Academic and University-affiliated community Hospitals. Ann Surg. https://doi.org/10.1097/SLA. 0000000000004642

Publisher's note Springer Nature remains neutral with regard to jurisdictional claims in published maps and institutional affiliations. 